\title{
Unplanned admission after gastrectomy as a consequence of fast-track surgery: a comparative risk analysis
}

\author{
Michitaka Honda $^{1} \cdot$ Naoki Hiki $^{1} \cdot$ Souya Nunobe ${ }^{1} \cdot$ Manabu Ohashi $^{1} \cdot$ \\ Shinji Mine $^{1} \cdot$ Masayuki Watanabe $^{1} \cdot$ Satoshi Kamiya $^{1} \cdot$ Tomoyuki Irino $^{1} \cdot$ \\ Takeshi Sano $^{1} \cdot$ Toshiharu Yamaguchi $^{1}$
}

Received: 31 January 2015/ Accepted: 28 September 2015/Published online: 7 October 2015

(c) The International Gastric Cancer Association and The Japanese Gastric Cancer Association 2015

\begin{abstract}
Background The objectives of this study were to evaluate the incidence of and the risk factors for readmission after gastrectomy. Our hypothesis was that early discharge may be related to an increase in the incidence of readmission. Methods This was a retrospective, single-center, observational study of 1442 patients who underwent gastrectomy for stage I gastric cancer. The main outcome was the incidence of early readmission after gastrectomy, which was defined as an admission within 6 months after the first discharge day. A stepwise logistic regression analysis was conducted to identify the risk factors for readmission after gastrectomy.

Results The surgical procedures performed were total gastrectomy in 217 patients (15.0\%), distal gastrectomy in 845 patients $(58.6 \%)$, pylorus-preserving gastrectomy in 342 patients $(23.7 \%)$, and proximal gastrectomy in 37 patients $(2.6 \%)$. The median hospital stay was 11 days, and there were 63 readmissions for a total of 56 patients (3.8\%). The main reasons for readmission were poor food intake in 14 patients $(22.2 \%)$, anastomotic stricture in nine patients $(14.3 \%)$, small bowel obstruction in eight patients $(12.7 \%)$, and an abdominal distension in seven patients $(11.1 \%)$. Endoscopic balloon dilation was performed for the nine patients with anastomotic stricture, drainage was needed for the four patients with an intra-abdominal abscess, and laparotomy was performed for one patient
\end{abstract}

Naoki Hiki

naoki.hiki@jfcr.or.jp

1 Department of Gastroenterological Surgery, Gastroenterological Center, Cancer Institute Hospital, Japanese Foundation for Cancer Research, 3-10-6 Ariake, Koto-ku, Tokyo 135-8550, Japan with adhesion-associated bowel obstruction. The long hospitalization during the primary admission and patients who underwent total gastrectomy were risk factors for readmission.

Conclusions Patients with a long hospital stay after gastrectomy are at an increased risk of early readmission, which was likely related to the incidence of severe sequelae specific to gastrectomy.

Keywords Gastric cancer - Surgery · Readmission · Risk factor

\section{Introduction}

Perioperative management reflecting concepts such as fast-track surgery and enhanced recovery after surgery has recently been used for gastric cancer surgery [1-3], These interventions are expected to enhance recovery of intestinal mobility, prevent the incidence of postoperative complications such as respiratory disease and thrombosis, and contribute to reducing the length of postoperative hospitalization and medical costs [2, 4-6], ultimately leading to patient satisfaction. On the other hand, there is concern about the association between impetuous use of such management and patient risk. Indeed, a relationship between a decrease in the length of postoperative hospital stay and an increased rate of readmission has been reported in colorectal cancer patients in the USA [7]. In addition, the same study indicated that there was a high incidence of in-hospital mortality among patients who were readmitted [7]. A shortened hospital stay may therefore be a disadvantage, and it is necessary to survey the readmission rate as an essential quality indicator to evaluate fast-track protocols. 
What should be considered a specific problem for patients who undergo gastrectomy is that various postoperative symptoms that often lead to eating problems, malnutrition, and weight loss during the early postoperative period [8-10]. Besides, these consequences could eventually affect the prognosis of patients through decreasing their tolerance to evidence-based postoperative chemotherapy [11]. Although setting an appropriate period of hospitalization is therefore required after gastric surgery, previous studies have provided few data about the rate of readmission after the first discharge following gastrectomy. In the USA, the incidence of and risk factors for readmission after various type of surgery have been published on the basis of large-scale data form the American College of Surgeons National Surgical Quality Improvement Program [12]. Unfortunately, however, patients who underwent gastrectomy were not included in the study.

We have conducted a retrospective single-institution survey to evaluate the hypothesis that early discharge reflecting the recent trend in perioperative care causes an increase in the incidence of readmission of postgastrectomy patients. Considering the adverse effects of gastrectomy generally last longer than for other types of surgery, early readmission in this study was defined as within 6 months postoperatively, which is relatively longer than in previous related studies. In addition, only patients with stage I gastric cancer were included in order to avoid the influence of readmission due to cancer recurrence. These results will help to decide whether further attempts to apply fast-track protocols for patients after gastrectomy can be justified.

\section{Methods}

\section{Patients}

Patients who underwent gastrectomy for gastric cancer from January 2007 to December 2011 were identified in the database of the Cancer Institute Hospital, Japanese Foundation for Cancer Research, Tokyo, Japan. To exclude the potential adverse effects of adjuvant chemotherapy or the diagnosis or treatment of recurrence, only patients with pathological stage I (Union for International Cancer Control/American Joint Committee on Cancer TNM classification, seventh edition [13]) disease (who are not treated with chemotherapy) were enrolled in this study. All patients who were confirmed to be alive at 12 months postoperatively were enrolled.

The items surveyed were the patients' characteristics, tumor profile, surgical information, and readmission data. The patient characteristics evaluated included the age, sex, body mass index, the risk grade of the American
Society of Anesthesiologists, and any comorbidities. The tumor profile included the pathological $\mathrm{T}$ and $\mathrm{N}$ factors and histological findings, and the surgical information included the kind of procedure and reconstruction performed, the length of the operation, the amount of blood lost, and any postoperative complications. This study was approved by the Institutional Review Board of our institute.

\section{Postoperative care and clinical path}

The clinical path was revised in September 2007. Before that point, patients were allowed to drink water from postoperative day (POD) 2 and to eat a soft diet from POD 4. On the basis of the results of blood tests and X-rays performed on POD 9, the physicians decided when the patient could be discharged. After September 2007, the patients started drinking water from POD 1 and eating a soft diet from POD 3. The decision regarding discharge was made on POD 7 unless postoperative complications were present. On the basis of the patient's request, discharge could be postponed for 7 days at most. The postoperative complications were recorded, and the degree was determined on the basis of the Clavien-Dindo classification [14], and discharge was postponed to treat complications as needed.

\section{Outcome measures and statistical analysis}

The main outcome of this study was the incidence of unplanned readmission after gastrectomy. Readmission was defined as an admission within 6 months after the first discharge day in this study, except for treatment for another disease. Aside from the checking of the medical records, a telephone survey at the patients' local clinic or hospitals was performed for who did not undergo periodic follow-up in our hospital. The reasons for readmission, the treatment(s) administered, and the length of hospitalization were investigated. To focus on the relationship between shortening the postoperative hospital stay and increasing the risk of readmission, the length of postoperative hospitalization was divided into three categories by a cut-off level (the mean \pm half the standard deviation), and the proportion of readmissions was compared for each category. Furthermore, a stepwise logistic regression analysis was performed to consider the influence of other confounding factors; the incidence of readmission was considered to be the dependent variable, and the items surveyed that had a possibility of being confounding factors were identified by the univariate analyses. The chisquare test for dichotomous variables and the $t$ test for continuous variables were performed for the univariate analyses. 


\section{Results}

\section{Study population}

A total of 1461 patients were identified who underwent surgery for gastric cancer during the study period. A total of 1442 patients were finally enrolled in this study, because one intrahospital death and 18 untraceable patients were excluded from the analysis. The patients' characteristics are shown in Table 1 . The median age was 66 years, and there were 962 male patients $(66.7 \%)$ in the all patients. The procedures performed were total gastrectomy in 217 patients $(15.0 \%)$, distal gastrectomy in 845 patients $(58.6 \%)$, pylorus-preserving gastrectomy in 342 patients $(23.7 \%)$, and proximal gastrectomy in 37 patients $(2.6 \%)$. There were 63 readmissions for a total of 56 patients. These patients were defined as the early readmission group (ERG).

The perioperative information is shown in Table 2 . Significantly more patients underwent total gastrectomy in the ERG. The postoperative hospital stay of the primary admission was prolonged in the ERG, and the incidence of postoperative complications was high in the ERG. The reasons for readmission and the timing of the readmission are shown in Table 3. Although there were many reasons for readmission, the main reasons were poor food intake (in 14 patients; $22.2 \%$ ), anastomotic stricture (in nine patients; 14.3\%), small bowel obstruction (in eight patients; $12.7 \%$ ), and abdominal distension (in seven patients; $11.1 \%$ ). The median length of hospitalization for readmission was 12 days (range $3-59)$. Eight of the patients $(88.9 \%)$ with anastomotic stricture underwent total gastrectomy and Roux-en-Y reconstruction, and endoscopic balloon dilation was performed. Percutaneous drainage was needed for the four patients with an intra-abdominal abscess, and laparotomy was performed for one patient with adhesion-related bowel obstruction. The mortality rate was zero in this series.

Table 1 The patients' characteristics

\begin{tabular}{|c|c|c|c|}
\hline & Non-ERG $(n=1386 ; 96.2 \%)$ & ERG $(n=56 ; 3.8 \%)$ & $p$ \\
\hline \multicolumn{4}{|l|}{ Age (years) } \\
\hline Median & 68 & 63 & \multirow[t]{2}{*}{0.0013} \\
\hline Range & $25-95$ & $29-88$ & \\
\hline \multicolumn{4}{|l|}{ Sex } \\
\hline Male & $917(66.2 \%)$ & $45(80.4 \%)$ & \multirow[t]{2}{*}{0.027} \\
\hline Female & $469(33.8 \%)$ & $11(19.6 \%)$ & \\
\hline BMI $\left(\mathrm{kg} / \mathrm{m}^{2}\right)^{\mathrm{a}}$ & $22.6 \pm 3.2$ & $22.8 \pm 3.6$ & 0.569 \\
\hline \multicolumn{4}{|c|}{ ASA physical status classification system score } \\
\hline 1 & $983(70.9 \%)$ & $35(62.5 \%)$ & \multirow[t]{3}{*}{0.387} \\
\hline 2 & $370(26.7 \%)$ & $19(33.9 \%)$ & \\
\hline 3 & $33(2.4 \%)$ & $2(3.6 \%)$ & \\
\hline \multicolumn{4}{|l|}{ Comorbidities } \\
\hline Hypertension & $217(15.7 \%)$ & $10(17.9 \%)$ & 0.142 \\
\hline Diabetes & $102(7.4 \%)$ & $4(7.1 \%)$ & 0.882 \\
\hline Cardiovascular & $51(3.8 \%)$ & $1(1.8 \%)$ & 0.497 \\
\hline Respiratory & $35(2.5 \%)$ & $4(7.1 \%)$ & 0.021 \\
\hline Anemia & $14(1.0 \%)$ & $0(0 \%)$ & 0.508 \\
\hline \multicolumn{4}{|l|}{ TNM stage $^{\mathrm{b}}$} \\
\hline IA & $1072(77.3 \%)$ & $39(69.6 \%)$ & \multirow[t]{2}{*}{0.430} \\
\hline IB & $370(26.7 \%)$ & $17(30.4 \%)$ & \\
\hline \multicolumn{4}{|l|}{ Histological type } \\
\hline Well-differentiated adenocarcinoma & $582(42.0 \%)$ & $24(42.9 \%)$ & \multirow[t]{3}{*}{0.802} \\
\hline Poorly differentiated adenocarcinoma & $786(56.7 \%)$ & $31(55.4 \%)$ & \\
\hline Others & $18(1.3 \%)$ & $1(1.7 \%)$ & \\
\hline
\end{tabular}

$A S A$ American Society of Anesthesiologists, $B M I$ body mass index, $E R G$ early readmission group

${ }^{\text {a }}$ Mean \pm standard deviation

${ }^{\mathrm{b}}$ Union for International Cancer Control/American Joint Committee on Cancer classification, seventh edition 
Table 2 The surgical procedures and outcomes

\begin{tabular}{|c|c|c|c|}
\hline & Non-ERG $(n=1386)$ & ERG $(n=56)$ & $p$ \\
\hline \multicolumn{4}{|l|}{ Procedure } \\
\hline TG & $193(13.9 \%)$ & $24(42.9 \%)$ & \multirow[t]{5}{*}{$0.000 *$} \\
\hline DG & $822(59.3 \%)$ & $23(41.1 \%)$ & \\
\hline B-1/R-Y & $370 / 452$ & $19 / 4$ & \\
\hline PPG & $336(24.2 \%)$ & $6(10.7 \%)$ & \\
\hline PG & $34(2.5 \%)$ & $3(5.4 \%)$ & \\
\hline \multicolumn{4}{|l|}{ Lymph node dissection } \\
\hline $\mathrm{D} 1+$ & $1055(76.1 \%)$ & $37(66.1 \%)$ & \multirow[t]{2}{*}{0.421} \\
\hline D2 & $331(23.9 \%)$ & $19(33.9 \%)$ & \\
\hline Laparoscopic surgery & $275(19.8 \%)$ & $16(28.6 \%)$ & 0.277 \\
\hline Length of operation $(\min )^{\mathrm{a}}$ & $236 \pm 63.0$ & $262 \pm 88.4$ & $0.009 *$ \\
\hline Blood loss $(\mathrm{ml})^{\mathrm{a}}$ & $86.7 \pm 137.7$ & $158.3 \pm 291.2$ & $0.008 *$ \\
\hline \multicolumn{4}{|c|}{ Postoperative hospital stay (days) } \\
\hline Median & 11 & 13 & \multirow[t]{2}{*}{$0.005^{*}$} \\
\hline Range & $7-252$ & $7-70$ & \\
\hline Postoperative complication(s) & $250(18.0 \%)$ & $20(35.7 \%)$ & $0.000 *$ \\
\hline Grade $\mathrm{I}^{\mathrm{b}}$ & $54(3.9 \%)$ & $4(7.1 \%)$ & \\
\hline Grade II $^{\mathrm{b}}$ & $167(12.0 \%)$ & $9(16.1 \%)$ & \\
\hline Grade III or greater $^{\mathrm{b}}$ & $29(11.6 \%)$ & $7(12.5 \%)$ & \\
\hline Reoperation & $7(0.5 \%)$ & $1(1.8 \%)$ & \\
\hline Preoperative ESD & $163(11.8 \%)$ & $6(10.7 \%)$ & \\
\hline
\end{tabular}

$B-1$ Billroth I reconstruction, $D G$ distal gastrectomy, $E R G$ early readmission group, ESD endoscopic submucosal dissection, $P G$ proximal gastrectomy, $P P G$ pylorus-preserving gastrectomy, $R-Y$ Roux-en-Y reconstruction, $T G$ total gastrectomy

* Significant difference

${ }^{\text {a }}$ Mean \pm standard deviation

b Clavien-Dindo classification

\begin{tabular}{llll}
\hline & $\leq 30$ days $(n=21)$ & $31-90$ days $(n=20)$ & $91-180$ days $(n=22)$ \\
\hline Malnutrition & $2(9.5 \%)$ & $4(20 \%)$ & $8(36.3 \%)$ \\
Anastomotic stricture & $3(14.3 \%)$ & $2(10 \%)$ & $4(18.2 \%)$ \\
Small bowel obstruction & $1(4.8)$ & $4(20 \%)$ & $3(13.6 \%)$ \\
Abdominal distention & $2(9.5 \%)$ & $3(15 \%)$ & $2(9.1 \%)$ \\
Enteritis & $2(9.5 \%)$ & $2(10 \%)$ & $2(9.1 \%)$ \\
Fever & $3(14.3 \%)$ & $2(10 \%)$ & 0 \\
Wound infection & $5(23.8 \%)$ & 0 & 0 \\
Abdominal abscess & $3(14.3 \%)$ & $1(5 \%)$ & 0 \\
Esophagitis & 0 & $1(5 \%)$ & $2(9.1 \%)$ \\
Cholecystitis & 0 & $1(5 \%)$ & $1(4.5 \%)$ \\
\hline
\end{tabular}

Table 3 The reasons for readmission and the timing of readmission after primary discharge

\section{Evaluation of the risk factors for unplanned readmission}

Figure 1 shows the relationship between the postoperative hospital stay during the primary admission and the incidence of readmission. The length of postoperative hospital stay was divided into three groups: short (less than 10 days), moderate (10-19 days), and long (more than 19 days). The proportions of readmissions in these groups were 2.02, 2.16 and $8.51 \%$, respectively; the incidence of readmission was higher in the long hospitalization group than in the other two groups. A logistic regression analysis isolated two factors as independent risk factors: total gastrectomy and a long hospitalization during the primary admission (Table 4). The Hosmer-Lemeshow goodness-of-fit test showed the high stability of this logistic model (Prob $>\chi^{2}=0.829$ ). 
Propotion of readmission

(\%)

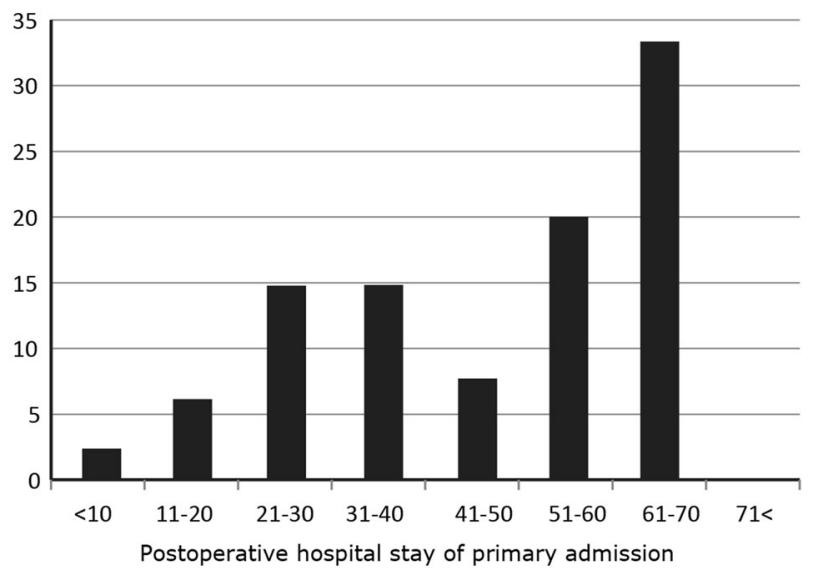

(days)

Fig. 1 The trend of the readmission and postoperative hospital stay at the primary admission. The proportion of readmissions was lower for patients with early discharge after surgery

\section{Discussion}

The duration of postoperative hospitalization is generally longer in Japan than in Western countries because the medical insurance system in Japan allows patients to stay in hospital with a low financial burden. The mean length of hospitalization after gastrectomy was reported to be 11-15 days [15-17]. Considering the fact that fast-track surgery has recently been accepted throughout the world $[18,19]$, we decided in September 2010 that most patients who undergo gastrectomy in our institute can be discharged on POD 7, whereas this was done on POD 9 before June 2010. However, some researchers have been emphasizing concerns about the relationship between the increasing incidence of readmission and early discharge after colorectal surgery $[7,20]$. Few studies have investigated the relationship between a shorter hospital stay and increased incidence of readmission among gastric cancer patients; therefore, we conducted the present study to evaluate the hypothesis that shortening the hospital stay after gastrectomy increases the risk of readmission. As a result, we found that shortening of the primary hospital stay was not a risk factor, whereas total gastrectomy and a long hospital stay after surgery were risk factors for readmission.

Recently, Merkow et al. [12] reported an association between readmission after various surgical procedures and the patients' or hospital's characteristics using a large-scale dataset: 498,875 patients who underwent bariatric surgery, colectomy or proctectomy, ventral hernia repair, hysterectomy, or arthroplasty or vascular bypass from 346 American College of Surgeons National Surgical Quality Improvement Program hospitals. Although gastrectomy was not included, the incidence of 30-day unplanned readmission was $5.7 \%$, and the main reasons were surgical site infection, obstruction or ileus, bleeding, or pulmonary events. Various patient characteristics, such as older age, male sex, smoking, obesity, various comorbidities, or postoperative complications, were associated with readmission. There were no data on the length of postoperative hospitalization at the primary admission. In addition, new complications after discharge, which were not associated with the complications during the initial admission, occurred in most of the patients who required readmission. In the present study, we focused on investigating the characteristics of unplanned readmission after gastrectomy. According to the results of the logistic regression model, the risk of readmission was not correlated with the incidence of postoperative complications; however, it was correlated with a long hospital stay at the primary admission. Namely, postoperative sequelae, such as nausea, heartburn, diarrhea, abdominal distention, or dumping syndrome were common in patients who have undergone gastrectomy $[9,10,21]$, and a long hospitalization may be associated with these postoperative symptoms, followed by difficulty in eating, rather than the treatment of
Table 4 The results of the analyses of potential risk factors for readmission after gastrectomy

\begin{tabular}{lllll}
\hline & Odds ratio & SE & $p$ & $95 \%$ CI \\
\hline Age ( $\leq 75$ years) & 1.663 & 0.618 & 0.171 & $0.803-3.443$ \\
Sex & 0.535 & 0.232 & 0.150 & $0.228-1.253$ \\
Respiratory disease & 2.771 & 1.654 & 0.088 & $0.860-8.925$ \\
TG/others & 3.423 & 1.281 & 0.001 & $1.644-7.130$ \\
Length of hospital stay & & & & \\
$\quad$ Short (compared with medium) & 1.004 & 0.563 & 0.994 & $0.334-3.016$ \\
$\quad$ Long (compared with medium) & 3.050 & 1.256 & $0.007 *$ & $1.361-6.837$ \\
Complication(s) & 1.413 & 0.554 & 0.379 & $0.654-3.051$ \\
Blood loss & 0.910 & 0.551 & 0.876 & $0.279-2.978$ \\
Length of operation & 1.324 & 0.532 & 0.485 & $0.602-2.908$ \\
\hline
\end{tabular}

$C I$ confidence interval, $S E$ standard error, $T G$ total gastrectomy

* Significant difference 
the postoperative complications. In particular, it is necessary to perform periodic follow-up studies in clinics after total gastrectomy in order to assess patients' abdominal symptoms and eating conditions and to intervene in order to prevent malnutrition. A previous report described that $80 \%$ of unexpected readmissions occurred within 30 days, and $50 \%$ of these were within 7 days after discharge in colorectal cancer patients [7, 22]. However, regarding specific gastric surgery findings, our study revealed that the incidence of readmissions occurred continuously for up to 6 months postoperatively, because the commonest reasons were malnutrition caused by severe sequelae after gastrectomy.

There are several limitations associated with this study. First, the subjects were limited to those with pathological stage I gastric cancer in order to exclude readmission according to the diagnosis or treatment of recurrence and to eliminate the effects of adjuvant chemotherapy on the risk of readmission. The association of the effects of chemotherapy should also be investigated in future investigations. Second, the decision to discharge or readmit patients was more or less influenced by the patients' preferences and their social problems, which were difficult to measure. However, such outcomes would be notable from the perspective of the patient-centered outcomes.

In conclusion, it is thought that discharge on PODs 7-9 after gastrectomy is not a risk factor for readmission compared with discharge on POD 10 or later. Patients with a long hospital stay after surgery or who underwent total gastrectomy had an increased risk of readmission, which was likely related to the incidence of severe sequelae following surgery and subsequent malnutrition.

\section{References}

1. Kim JW, Kim WS, Cheong JH, Hyung WJ, Choi SH, Noh SH. Safety and efficacy of fast-track surgery in laparoscopic distal gastrectomy for gastric cancer: a randomized clinical trial. World J Surg. 2012;36:2879-87.

2. Yamada T, Hayashi T, Aoyama T, Shirai J, Fujikawa H, Cho H, et al. Feasibility of enhanced recovery after surgery in gastric surgery: a retrospective study. BMC Surg. 2014;14:41.

3. Sugisawa N, Tokunaga M, Makuuchi R, Miki Y, Tanizawa Y, Bando E, et al. A phase II study of an enhanced recovery after surgery protocol in gastric cancer surgery. Gastric Cancer. 2015. doi:10.1007/s10120-015-0528-6.

4. Gianotti L, Nespoli L, Torselli L, Panelli M, Nespoli A. Safety, feasibility, and tolerance of early oral feeding after colorectal resection outside an enhanced recovery after surgery (ERAS) program. Int J Color Dis. 2011;26:747-53.

5. Ren L, Zhu D, Wei Y, Pan X, Liang L, Xu J, et al. Enhanced recovery after surgery (ERAS) program attenuates stress and accelerates recovery in patients after radical resection for colorectal cancer: a prospective randomized controlled trial. World J Surg. 2012;36:407-14.
6. Rossi G, Vaccarezza H, Vaccaro CA, Mentz RE, Im V, Alvarez A, et al. Two-day hospital stay after laparoscopic colorectal surgery under an enhanced recovery after surgery (ERAS) pathway. World J Surg. 2013;37:2483-9.

7. Schneider EB, Hyder O, Brooke BS, Efron J, Cameron JL, Edil $\mathrm{BH}$, et al. Patient readmission and mortality after colorectal surgery for colon cancer: impact of length of stay relative to other clinical factors. J Am Coll Surg. 2012;214:390-8; discussion 398-9.

8. Honda M, Wakita T, Onishi Y, Nunobe S, Hiki N, Miura A, et al. Development and validation of a symptom scale to evaluate postoperative patients with esophagogastric cancer. J Am Coll Surg. 2014;219:895-903.

9. Karanicolas PJ, Graham D, Gonen M, Strong VE, Brennan MF, Coit DG. Quality of life after gastrectomy for adenocarcinoma: a prospective cohort study. Ann Surg. 2013;257:1039-46.

10. Mine S, Sano T, Tsutsumi K, Murakami Y, Ehara K, Saka M, et al. Large-scale investigation into dumping syndrome after gastrectomy for gastric cancer. J Am Coll Surg. 2010;211: 628-36.

11. Aoyama T, Kawabe T, Fujikawa H, Hayashi T, Yamada T, Tsuchida K, et al. Loss of lean body mass as an independent risk factor for continuation of S-1 adjuvant chemotherapy for gastric cancer. Ann Surg Oncol. 2015;22:2560-6.

12. Merkow RP, Ju MH, Chung JW, Hall BL, Cohen ME, Williams $\mathrm{MV}$, et al. Underlying reasons associated with hospital readmission following surgery in the United States. JAMA. 2015;313: 483-95.

13. Biondi A, Hyung WJ. Seventh edition of TNM classification for gastric cancer. J Clin Oncol. 2011;29:4338-9; author reply 4340-2.

14. Dindo D, Demartines N, Clavien PA. Classification of surgical complications: a new proposal with evaluation in a cohort of 6336 patients and results of a survey. Ann Surg. 2004;240: 205-13.

15. Kinoshita T, Shibasaki H, Oshiro T, Ooshiro M, Okazumi S, Katoh R. Comparison of laparoscopy-assisted and total laparoscopic Billroth-I gastrectomy for gastric cancer: a report of shortterm outcomes. Surg Endosc. 2011;25:1395-401.

16. Yasunaga H, Horiguchi H, Kuwabara K, Matsuda S, Fushimi K, Hashimoto $\mathrm{H}$, et al. Outcomes after laparoscopic or open distal gastrectomy for early-stage gastric cancer: a propensity-matched analysis. Ann Surg. 2013;257:640-6.

17. Katai H, Sasako M, Fukuda H, Nakamura K, Hiki N, Saka M, et al. Safety and feasibility of laparoscopy-assisted distal gastrectomy with suprapancreatic nodal dissection for clinical stage I gastric cancer: a multicenter phase II trial (JCOG 0703). Gastric Cancer. 2010;13:238-44.

18. Spanjersberg WR, Reurings J, Keus F, van Laarhoven CJ. Fast track surgery versus conventional recovery strategies for colorectal surgery. Cochrane Database Syst Rev. 2011;(2): CD007635.

19. Wind J, Polle SW, Fung Kon Jin PH, Dejong CH, von Meyenfeldt MF, Ubbink DT, et al. Systematic review of enhanced recovery programmes in colonic surgery. Br J Surg. 2006;93: $800-9$.

20. Jakobsen DH, Sonne E, Andreasen J, Kehlet H. Convalescence after colonic surgery with fast-track vs conventional care. Colorectal Dis. 2006;8:683-7.

21. Nunobe S, Sasako M, Saka M, Fukagawa T, Katai H, Sano T. Symptom evaluation of long-term postoperative outcomes after pylorus-preserving gastrectomy for early gastric cancer. Gastric Cancer. 2007;10:167-72.

22. Azimuddin K, Rosen L, Reed JF 3rd, Stasik JJ, Riether RD, Khubchandani IT. Readmissions after colorectal surgery cannot be predicted. Dis Colon Rectum. 2001;44:942-6. 